\title{
Recent Development and Application of a New Safety Analysis Code for Fusion Reactors
}

\author{
*Brad J. Merrill, Paul W. Humrickhouse and Masashi Shimada \\ Idaho National Laboratory, PO Box 1625, Idaho Falls, ID, 83415, USA
}

\begin{abstract}
This paper describes the recent progress made in the development of two codes for fusion reactor safety assessments at the Idaho National Laboratory (INL): MELCOR for fusion and the Tritium Migration Analysis Program (TMAP). During the ITER engineering design activity (EDA), the INL Fusion Safety Program (FSP) modified the MELCOR 1.8.2 code for fusion applications to perform ITER thermal hydraulic safety analyses. Because MELCOR has undergone many improvements at SNL-NM since version 1.8.2 was released, the INL FSP recently imported these same fusion modifications into the MELCOR 1.8.6 code, along with the multiple fluids modifications of MELCOR 1.8.5 for fusion used in US advanced fusion reactor design studies. TMAP has also been under development for several decades at the INL by the FSP. TMAP treats multi-specie surface absorption and diffusion in composite materials with dislocation traps, plus the movement of these species from room to room by fluid flow within a given facility. Recently, TMAP was updated to consider multiple trap site types to allow the simulation of experimental data from neutron irradiated tungsten. The natural development path for both of these codes is to merge their capabilities into one computer code to provide a more comprehensive safety tool for analyzing accidents in fusion reactors. In this paper we detail recent developments in this area and the application of this new capability to a DEMO relevant water-cooled tungsten armored divertor.
\end{abstract}

Keywords: fusion safety, MELCOR, LOCA, TMAP, tritium transport.

\section{Introduction}

This paper describes the recent progress made in the development of two computer codes for fusion reactor safety assessments at the Idaho National Laboratory (INL): MELCOR for fusion and the Tritium Migration Analysis Program (TMAP). During the ITER engineering design activity (EDA), the INL Fusion Safety Program (FSP) modified the MELCOR 1.8.2 computer code for fusion applications to perform ITER thermal hydraulic safety analyses [1]. MELCOR, a computer code that is being developed by Sandia National Laboratory in New Mexico (SNL-NM), was chosen because of its ability to predict thermal-hydraulic transients while self-consistently accounting for aerosol transport in nuclear facilities and reactor cooling systems [2]. Because activated dust, whether originating from liquid evaporation or plasma facing component (PFC) erosion, is a radioactive substance that must tracked during accidents, MELCOR's aerosol capability is uniquely suited for fusion safety analyses.

Since MELCOR has undergone many improvements at SNL-NM since version 1.8.2 was released, the INL FSP recently imported the modifications for ITER into the MELCOR 1.8.6 code [3]. For the improvement described in this paper, the multiple fluids capabilities of MELCOR 1.8.5 for fusion, used in US ARIES reactor design studies [4], was added to this version of the MELCOR 1.8.6 code.

TMAP has also been under development for several decades by the FSP at INL. TMAP treats multi-specie surface absorption and diffusion in composite materials with dislocation traps, plus the movement of these species from room to room by fluid flow within a given facility. Recently, TMAP was updated to consider

Brad.Merrill@inl.gov multiple trap site types to allow the simulation of experimental data from neutron irradiated tungsten [5].

Because both of these codes are used by the FSP for fusion reactor safety assessments, the natural development path is to merge their capabilities to provide a more comprehensive tool for analyzing accidents in fusion reactors. The significance of this development effort is that it addresses two deficiencies noted by users of MELCOR for fusion: 1) an accurate tritium transport capability for MELCOR and 2) a more realistic time-dependent fluid and heat transport capability for TMAP. In this paper we detail the progress of this development activity and the application of this new capability by predicting the tritium inventory and permeation rate in a tungsten armored PFC.

The following section of this paper contains an overview of the MELCOR for fusion code development history. A comparison between MELCOR 1.8.2 and 1.8.6 for fusion is also included in this section. Section 3 discusses recent modifications to the TMAP4 code for multiple trap sites and TMAP's inclusion in the MELCOR for fusion code. Section 4 presents the predictions of this new version of MELCOR for a DEMO relevant water-cooled tungsten armored divertor. The final section summarizes the results of these code improvements to date.

\section{Overview of MELCOR for fusion computer code development}

As mentioned in the introduction, the MELCOR 1.8.2 code was chosen for thermal hydraulic safety analysis application to the evolving ITER reactor design during the ITER EDA. This version of the code included oxidation models for beryllium, graphite and tungsten. Other changes were made to MECLOR 1.8.2, 
for example: 1) to the aerosol transport package to track aerosols in atmospheres other than air, a standard MELCOR assumption, which is important for tracking dust produced by plasma erosion of PFCs in a helium atmosphere, and 2) water freezing on cryogenic surfaces [1]. This version of the code was used in ITER's NonSite-specific Safety Reports (NSSR) 1\&2 and Generic Site Safety Report (GSSR). Recently, the MELCOR 1.8.2 for ITER code underwent a final verification and validation $(\mathrm{V} \& \mathrm{~V})$ process and was used by the ITER International Organization (IO) for ITER's "Rapport Preliminaire de Surete" (Report Preliminary on Safety RPrS) [6].

Starting in 1998, MELCOR development took a new direction at the INL, when the MELCOR 1.8.5 code was modified to accommodate working fluids other than water. The code was modified to accept the ATHENA/RELAP [7] property tables which included: $\mathrm{PbLi}$, FLiBe, Lithium, Helium, Nitrogen, Hydrogen and others. To make this change as simple as possible, the standard MELCOR water properties were replaced with those from ATHENA/RELAP because of the conflict in units between these properties sets. The standard MELCOR Equation of State is in CGS units, while those of ATHENA are in SI units. This version of MELCOR for fusion was used by the INL in safety analyses of a number of advanced fusion reactor concepts, the most recent of which is ARIES ACT [4].

In developing the most recent version of MELCOR 1.8.6 for fusion, the multiple fluids modifications were made to this version of the MELCOR code while leaving the standard MELCOR water properties intact. This approach has the advantage of combining both previous versions (e.g. 1.8.2 and 1.8.5) in to a single code. In the absence of an ATHENA fluid properties file, the code defaults to the standard MELCOR water properties, which are identical to the properties in the MELCOR 1.8.2 for ITER code. Another advantage of this new version is that its predictions have double precision accuracy, unlike the versions 1.8.2 and 1.8.5 for fusion which have only single precision accuracy. This version of the code (MELCOR 1.8.6 for fusion) was made available to the MELCOR user community through SNL-NM during August of 2015.

Before MELCOR 1.8.6 for fusion was released, the code underwent a standard benchmarking exercise. One of the test problems used for this exercise is a calculation that appears in ITER's GSSR, which is a large in-vessel loss-of-coolant accident (LOCA). Figs. 1 and 2 contain comparisons between the MELCOR 1.8.6 for fusion and MELCOR 1.8.2 for ITER codes for this accident. Fig. 1 contains the predicted vacuum vessel (VV) pressure comparison. Fig. 2 contains the predicted VV vapor temperature comparison. For these calculations, the invessel LOCA begins at $1000 \mathrm{~s}$. Prior to this time, the codes are establishing a steady state to initialize the system models. As can be seen, there is excellent agreement for these two parameters between these codes. Given the page limitation, only these comparisons will be presented here. A benchmarking report is presently in preparation to provide a more detailed comparison.

\section{Overview of the TMAP computer code development}

The first official release of version 1 of the Tritium Migration Analysis Program (TMAP1) computer code was in 1988 [8]. TMAP4, a V\&V'd version of TMAP, was available in 1992 [9] and soon after was submitted to the Department of Energy's Energy Science and Technology Software Center (ESTSC) to be placed under configuration control. TMAP7 was available from the INL in 2005, which had the added capability of treating up to three dislocation traps [10]. Unfortunately

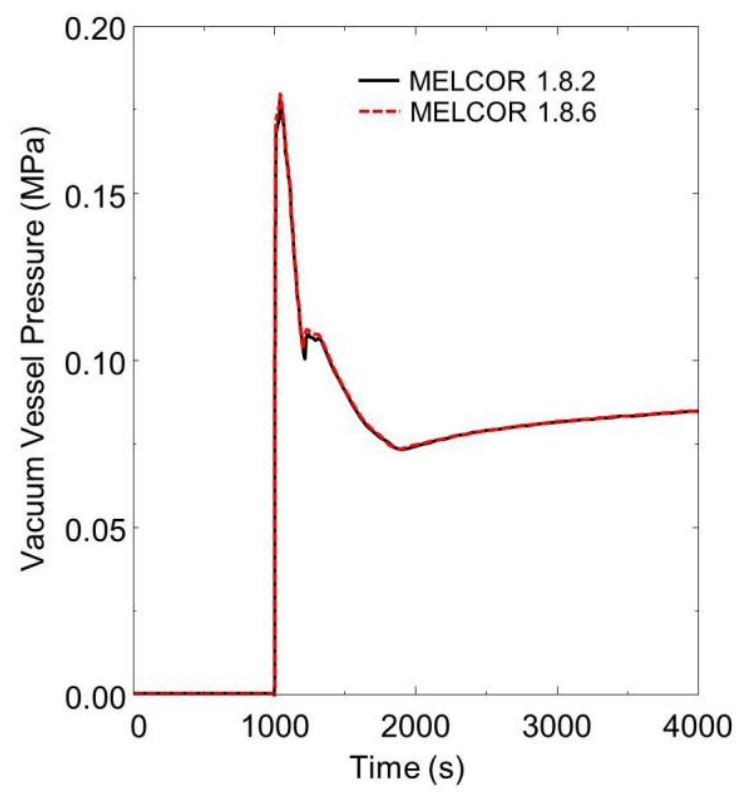

Fig. 1. Predicted VV pressure for two different versions of the MELCOR for fusion computer code.

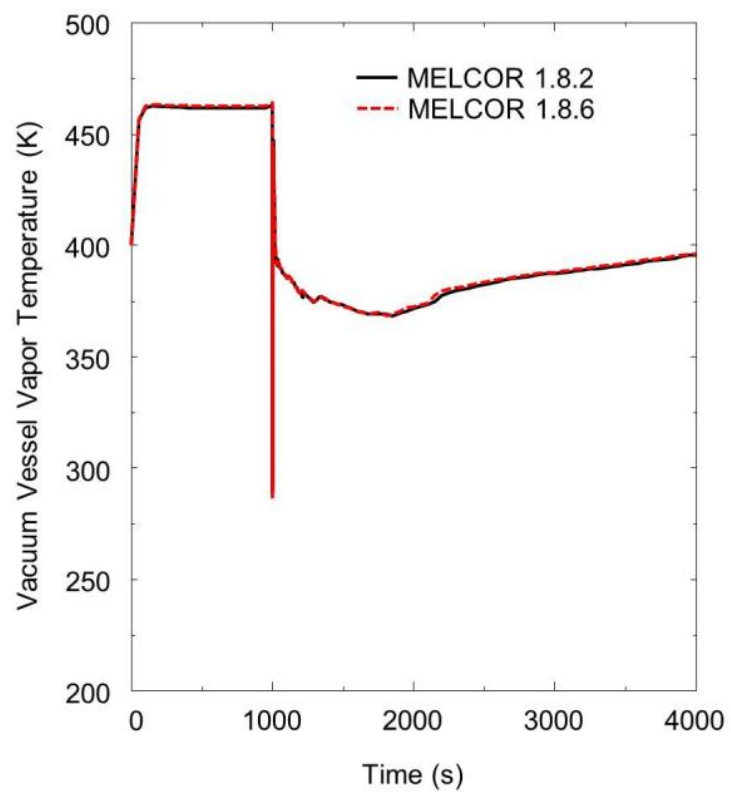

Fig. 2. Predicted VV vapor temperature for two different versions of the MELCOR for fusion computer code. 
this version of the code was not placed under configuration control. However, as mentioned in the introduction, recently the TMAP4 code was modified to include as many dislocation traps as the user desired [5].

The reason dislocation traps are important to tritium safety is that $14 \mathrm{MeV}$ neutrons generated by the fusion process create significant damage to the plasma facing component (PFC) material lattice by atomic displacements and elemental changes through transmutations [11]. The damage sites act as ideal traps for tritium implanted in the surface of a PFC by the plasma as it diffuses and is either released back into the $\mathrm{VV}$, or into the coolant at the opposite side of the PFC. One of the research goals of the US/Japan TITAN collaborative agreement was to experimentally determine the extent to which tritium would be trapped in neutron irradiated tungsten. Tungsten samples that that are $6 \mathrm{~mm}$ in diameter and $0.2 \mathrm{~mm}$ thick were irradiated to three different damage levels $(0.025,0.3$ and $2.4 \mathrm{dpa}$ ) in ORNL's High Flux Isotope Reactor (HFIR) at a temperature of $50-70{ }^{\circ} \mathrm{C}$ under this agreement.

After irradiation, these samples were exposed to deuterium plasmas in the Tritium Plasma Experiment (TPE) at INL's Safety and Tritium Applied Research (STAR) facility. TPE is a linear plasma device [12] capable of exposing target material to low-energy (100 $\mathrm{eV})$ deuterons at a plasma ion flux of up to $(5-7) \times 10^{21}$ $\mathrm{m}^{-2} \mathrm{~s}^{-1}$. For the irradiated tungsten samples the plasma was maintained until an ion fluence of $(5-7) \times 10^{25} \mathrm{~m}^{-2}$ was achieved for sample temperatures of 100, 200 and $500{ }^{\circ} \mathrm{C}$. After being exposed to deuterium plasmas in TPE, while being cooled at $200{ }^{\circ} \mathrm{C}$, several of the samples were shipped to the Ion Beam Laboratory at the University of Wisconsin, Madison for Nuclear Reaction Analysis (NRA) [12]. Fig. 3 contains the deuterium depth profiles determined by this diagnostic device.

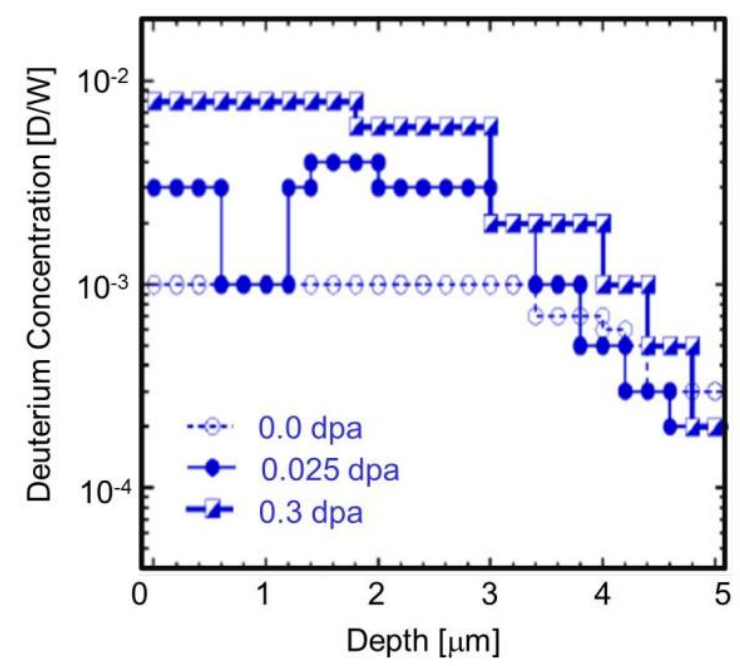

Fig. 3. Deuterium depth profiles for tungsten exposed to a TPE plasma at $200^{\circ} \mathrm{C}$ as measured by NRA.
The high deuterium concentrations measured in these samples are a likely indication that this deuterium resides in dislocation traps. According to Ref. [11], the concentration of traps in un-irradiated tungsten is about $1.5 \times 10^{-5}$ per tungsten atom. It was measured to be higher for the tungsten examined here at $\sim 1.0 \times 10^{-3}$ traps per tungsten atom (note 0 dpa data in Fig. 3).

After the NRA analysis, the samples were placed a Thermal Desorption System (TDS) at STAR, where they were heated under a controlled linear temperature change from 25 to $800{ }^{\circ} \mathrm{C}$. The off-gas from the samples was measured by this system's Residual Gas Analyzer (RGA). The TDS spectrum (released deuterium plotted against sample temperature) obtained for a $0.025 \mathrm{dpa}$ sample used in this study appears in Fig. 4.

This same TDS spectrum was analyzed by Ref. [5] with the TMAP4 computer code by applying six separate traps with trap densities and energies that were varied to match the TDS spectrum, with the proviso that the sum of the trap densities equaled the measured deuterium density of Fig. 3. At each TDS temperature along the spectrum, the modeled traps release their deuterium to provide the predicted result in Fig. 4. In order to get the correct deuterium inventory in the sample prior to TDS analysis, the same TMAP model was used to simulate the plasma $\mathrm{D}^{+}$ion implantation, diffusion, and trapping within the bulk of the tungsten sample during TPE operation.

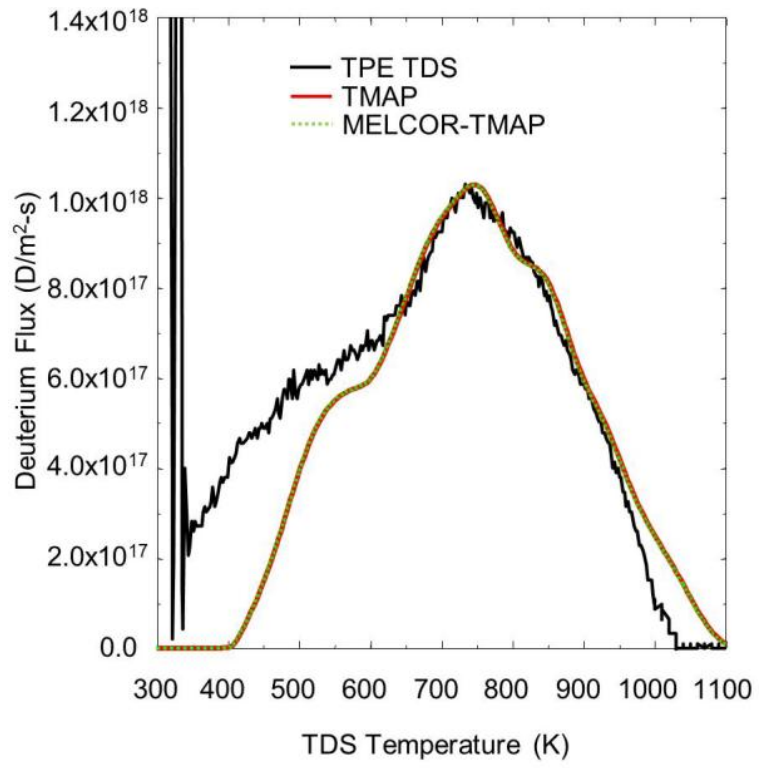

Fig. 4. Deuterium TDS spectrum and code comparisons for tungsten irradiated to $0.025 \mathrm{dpa}$ and exposed to a TPE plasma at a tungsten temperature of $200^{\circ} \mathrm{C}$.

As mentioned in the introduction, a goal of the present code development effort is to integrate the capabilities of TMAP in the MELCOR for fusion code. To this end, hydrogen species $(\mathrm{H}, \mathrm{D}$ and $\mathrm{T})$ material diffusion, trapping, and surface kinetics models from the TMAP code were imported into the MELCOR 1.8.6 for fusion code this year. The same TPE charging and TDS off-gassing transient was simulated with this new 
MELCOR for fusion code. The result also appears in Fig. 4, illustrating that integration has been successfully accomplished. This combined capability, although still under development and testing, represents a powerful, more self-consistent safety analysis tool for analyzing fusion reactor accidents. All of the material property data required for MELCOR to track tritium transport in the reactor and confinement building of a fusion facility can now be entered through the MELCOR user material input data options. This reduces the previous effort to build and execute a TMAP model, plus provides TMAP with control volume options through MELCOR that were not previously available to this code.

\section{The application of MELCOR to a simplified DEMO relevant water-cooled tungsten armored divertor}

In this section, the newly developed MELCOR for fusion code is used to simulate the retention and permeation of tritium in a simple tungsten divertor model exposed to assumed DEMO relevant divertor plasma and water cooling conditions. For this simulation, it was assumed that the plasma heat flux on the divertor surface is $10 \mathrm{MW} / \mathrm{m}^{2}$. For a plasma ion temperature of $150 \mathrm{eV}$ at surface of the tungsten, a 10 $\mathrm{MW} / \mathrm{m}^{2}$ heat flux translates into an implanted plasma ion flux of $\sim 5 \times 10^{23}$ ions $/ \mathrm{m}^{2}$-s, equally split between $\mathrm{T}$ and $\mathrm{D}$ ions.

A thickness for the tungsten armor of $8 \mathrm{~mm}$ was adopted for this simulation. The coolant side of the tungsten target was held fixed at $423 \mathrm{~K}\left(150{ }^{\circ} \mathrm{C}\right)$, with the surface temperature calculated by MELCOR to be $1000 \mathrm{~K}$ for the $10 \mathrm{MW} / \mathrm{m}^{2}$ plasma heat flux.

The boundary conditions, material properties, and trap specifications used in this analysis are those determined, or used, in the TPE experiment simulation of the previous section. TMAP's surface kinetics model of atomic recombination was applied at the plasma surface to form $\mathrm{T}_{2}$, DT and $\mathrm{D}_{2}$ which were released back into the plasma chamber and a zero $\mathrm{T}$ and $\mathrm{D}$ concentration at the surface applied on the water cooled surface.

First, the case of permeation without displacement induced trapping was examined. This was to determine if reasonable agreement could be obtained from a simple equation presented in Ref. [11] and the MELCORTMAP results. This equation (Eq. 3 in Ref. 11) gives the depth to which traps are filled in tungsten versus time. According to this equation, the time, $\mathrm{t}(\mathrm{s})$, required to completely fill the traps to a given depth, $\mathrm{x}(\mathrm{m})$, is:

$$
t=x^{2}\left(N_{t} / 2 D C_{s}\right) \quad(\text { when } x \ll \sqrt{D t})
$$

where $N_{t}$ is trap density $\left(\mathrm{m}^{-3}\right), D$ is the diffusivity of tritium in the tungsten $\left(\mathrm{m}^{2} / \mathrm{s}\right)$ and $C_{s}\left(\mathrm{~m}^{-3}\right)$ is the tritium surface concentration produced by the plasma, here calculated by MELCOR to be $\sim 3.5 \times 10^{23}\left(\mathrm{~m}^{-3}\right)$. The trap density was set to that stated by Ref. [11] of $1.5 \times$ $10^{-5}$ per tungsten atom, or $9.3 \times 10^{23}\left(\mathrm{~m}^{-3}\right)$. To allow the comparison to complete within a reasonable time, a uniform tungsten temperature of $600 \mathrm{~K}$ was applied. The time estimates by Eq. 1 to completely fill all the traps in an $8 \mathrm{~mm}$ thick tungsten armor is $\sim 220 \mathrm{~h}$ for these conditions.

Fig. 5 contains the MELCOR-TMAP prediction for this case, showing a complete filling of traps, seen as a sudden rise in the water side permeation rate at $\sim 240 \mathrm{~h}$. This prediction is in close agreement with Eq. 1 given that this approximate equation is only good for depths much less than $\sim 9 \mathrm{~mm}$ for these conditions. This figure also contains a MELCOR-TMAP prediction for unirradiated tungsten for the assumed DEMO conditions. For this case, the trap energy was changed from the arbitrarily high value of the previous case to a value of $1.04 \mathrm{eV}$ [11]. Because the traps near the plasma side do not saturate at these temperatures, the permeation rate shows a gradual rise that reaches equilibrium within $1000 \mathrm{~h}$.

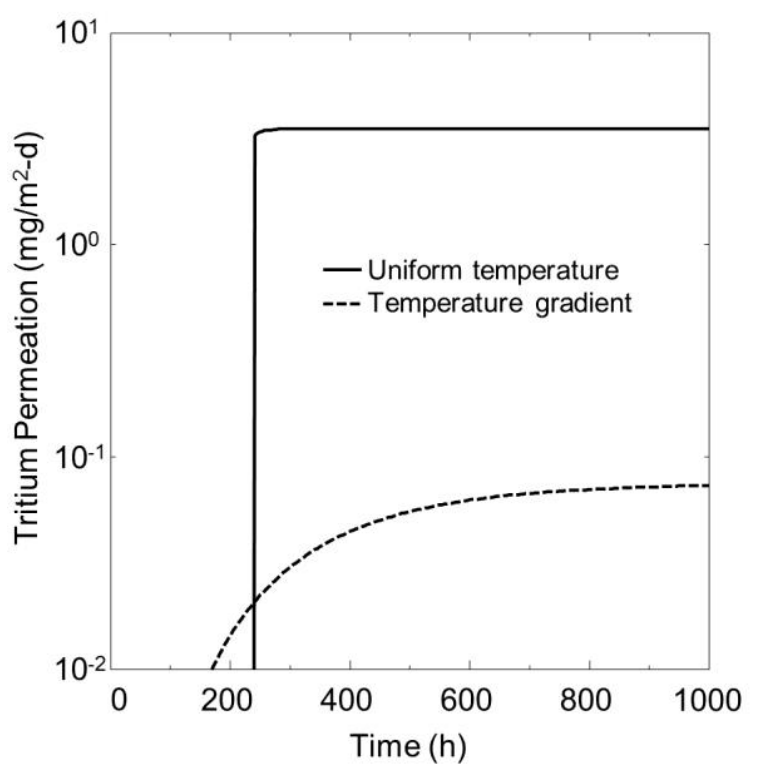

Fig. 5. Permeation rate into the coolant for un-irradiated tungsten test cases: a $600 \mathrm{~K}$ uniform temperature, and a temperature gradient from $1000 \mathrm{~K}$ to $423 \mathrm{~K}$.

Returning to the DEMO analysis, in order to examine the impact of neutron induced traps, a trap density must be adopted. The retention evident in Fig. 4 reflects an evolving trap site density that is not linear with irradiation damage (dpa). Because of material annealing, this damage depends not only on neutron fluence but irradiation temperature, and should also reach a saturation value past a certain level of damage. Here, a value of $1.0 \times 10^{-2}$ per tungsten atom was chosen, which bounds the data in Fig. 3.

While Eq. 1 is not applicable for temperature gradients and multiple trap sites, if an average tungsten temperature is assumed and this total trap density is used, the predicted time to achieve complete trap filling in the tungsten armor is estimated to be $\sim 60 \mathrm{y}$, given a MELCOR-TMAP predicted plasma surface concentration of $\sim 2.8 \times 10^{22}\left(\mathrm{~m}^{-3}\right)$. Fig. 6 contains the predicted tritium inventory for this case. The agreement 
between this prediction and that from Eq. 1 is most likely merely coincidental. In addition, because the divertor will be replaced after $\sim 2$ full power years (FPY) of operation [13], beyond two years the results of Fig. 6 become more academic in nature. At $2 \mathrm{FPY}$, the tritium inventory is $1.3 \mathrm{~g} / \mathrm{m}^{2}$.

The final case examined here deals with the question of whether or not there would be any permeation of tritium into the water for the assumed conditions. Ref. [14] calculates that the tungsten divertor would experience 3.2 dpa in 1 FPY of operation. For this case, it was assumed that the trap site density changed linearly with dpa, but saturated at $1.0 \times 10^{-2}$ per tungsten atom. The insert in Fig. 6 (expanded zone) shows the answer to this question. Basically, the production of dislocation trap sites proceeds at a rate that traps the implanted tritium before it can reach the coolant side of the tungsten armor, because the overall tritium inventory is identical to the case that assumes a constant trap site density after 0.2 y of divertor operation.

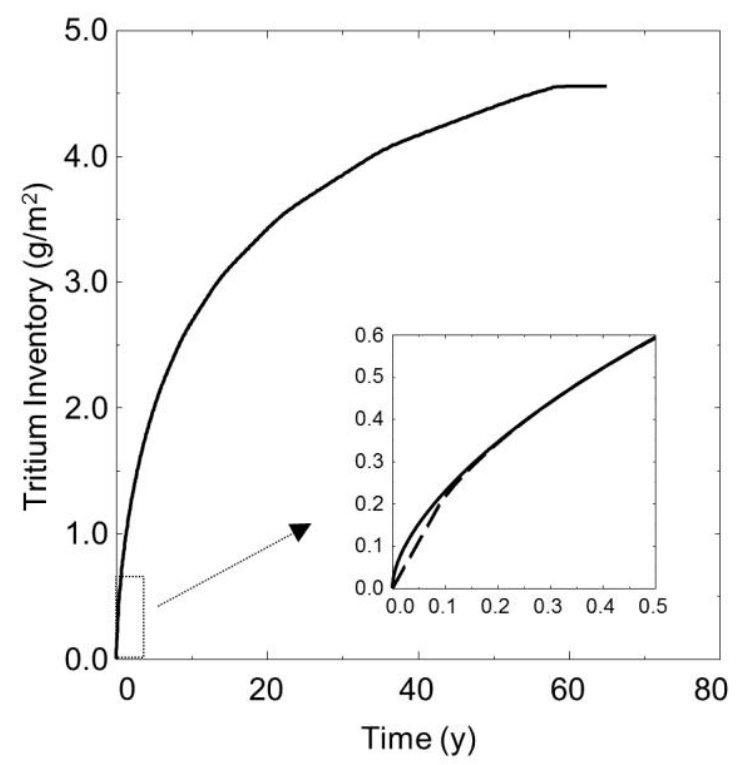

Fig. 6. Tritium inventory in a tungsten armored water cooled divertor.

\section{Summary}

In this paper, the development activities for a new version of MELCOR 1.8.6 for fusion that integrates the capabilities of the TMAP were presented. This new capability was applied to the problem of tritium retention and permeation in a water-cooled tungsten-armored divertor, based on a model that simulates the dislocation trapping data obtained from the US/JA TITAN collaborative agreement. The results presented illustrate the importance of this data to any safety assessment of a DEMO reactor that uses a tungsten divertor. However, more data on how dislocation traps evolve with irradiation temperature is needed to address this issue in more detail. Some of this data should be provided by the follow on agreement to TITAN called PHENIX.
In the near term, the development and validation of this version of MELCOR for fusion will continue at INL, with a planned release during calendar year 2018. When released, the user of this code will have a comprehensive tool for analyzing accidents in fusion reactors.

\section{Acknowledgments}

This material is based upon work supported by the U. S. Department of Energy Office of Science, Office of Fusion Energy Sciences, under the DOE Idaho Operations Office contract number DE-AC0705 ID 14517.

\section{References}

1. B. Merrill, R. Moore, S. Polkinghorne, and D. Petti, "Modifications to the MELCOR code for application in fusion accident analyses," Fusion Engineering and Design, 51-52 (2000) 555-563.

2. R. O. Gauntt, J. E. Cash, R. K. Cole, C. M. Erickson, L. L. Humphries, S. B. Rodriguez, and M. F. Young, MELCOR Computer Code Manuals, Nuclear Regulatory Commission Report, NUREG/CR-6119, Vol. 2, Rev. 3, September (2005).

3. B. Merrill, P. Humrickhouse, R. Moore, "A recent version of MELCOR for fusion safety applications," Fusion Engineering and Design, 85 (2010) 14791483.

4. P. W. Humrickhouse and B. J. Merrill, "ARIESACT1 Safety Design and Analysis," Fusion Science and Technology, vol. 67, pp. 167-178, January (2015).

5. B. J. Merrill, M. Shimada, P. W. Humrickhouse, "Simulating Tritium Retention in Tungsten with a Multiple Trap Model in the TMAP Code," Journal of Plasma Fusion Research SERIES, 10 (2013) 071.

6. S. Reyes, L. Topilski, N. Taylor, B. Merrill, L. Sponton, "Updated modeling of postulated accident scenarios in ITER," Fusion Science and Technology, 56 (2009) 789-793.

7. K. E. Carlson, P. A. Roth, V. H. Ransom, ATHENA Code Manual, Idaho National Laboratory Report, EGG-RTH-7397, September (1986).

8. B. J. Merrill, J. L. Jones, D. F. Holland, TMAP/MOD1: Tritium migration analysis program code description and user's manual, EGG-EP-7407, Idaho National Engineering Laboratory Report, April (1988).

9. G. R. Longhurst, D. F. Holland, J. L. Jones, B. J. Merrill, TMAP4 user manual, EGG-FSP-10315, Idaho National Engineering Laboratory Report, June (1992).

10.G. R. Longhurst, TMAP7: Tritium Migration Analysis Program: User Manual, INEEL/EXT-0402352, Idaho National Laboratory, Rev. 2 (2008).

11. W. R. Wampler, R. P. Doerner, "The influence of displacement damage on deuterium retention in tungsten exposed to plasma," Nuclear Fusion, 49, 11502 (2009).

12. M. Shimada, G Cao, T Otsuka, M Hara, M Kobayashi, Y Oya and Y Hatano, "Irradiation effect on deuterium behaviour in low-dose HFIR neutron- 
irradiated tungsten exposed to plasma," Nuclear Fusion, 55, 013008, (2015).

13.D. Maisonnier, et al., A Conceptual Study of Commercial Fusion Power Plants, EFDA(05)27/4.10, European Fusion Development Agreement Report, April (2005).

14.M.R. Gilbert, S.L. Dudarev, S. Zheng, L.W. Packer, J.-Ch. Sublet, "An integrated model for materials in a fusion power plant: transmutation, gas production, and helium embrittlement under neutron irradiation," Nuclear Fusion, 52, 083019, August (2012), 12pp. 